OPEN ACCESS

Edited by:

Jian-ye Zhang,

Guangzhou Medical University, China

Reviewed by:

Rufeng Wang,

Shanghai University of Traditional

Chinese Medicine, China

Jinfeng Zhang,

Beijing Institute of Technology, China

*Correspondence:

Min $\mathrm{Hu}$

humin2019@jnu.edu.cn

Lijuan Deng

ljdeng@jnu.edu.cn

tThese authors have contributed equally to this work

Specialty section:

This article was submitted to

Cellular Biochemistry,

a section of the journal

Frontiers in Cell and Developmental

Biology

Received: 09 October 2021 Accepted: 26 November 2021

Published: 13 December 2021

Citation:

Huang $M$, Lei $Y$, Zhong $Y$, Chung $C$, Wang M, Hu M and Deng L (2021) New

Insights Into the Regulatory Roles of

Extracellular Vesicles in Tumor

Angiogenesis and Their

Clinical Implications.

Front. Cell Dev. Biol. 9:791882.

doi: 10.3389/fcell.2021.791882

\section{New Insights Into the Regulatory Roles of Extracellular Vesicles in Tumor Angiogenesis and Their Clinical Implications}

\author{
Maohua Huang ${ }^{1,2 t}$, Yuhe $\mathrm{Lei}^{3 \dagger}$, Yinqin Zhong ${ }^{3 \dagger}$, Chiwing Chung ${ }^{1}$, Mei Wang ${ }^{1}$, Min $\mathrm{Hu}^{4 *}$ and \\ Lijuan Deng ${ }^{1 *}$
}

${ }^{1}$ Formula Pattern Research Center, School of Traditional Chinese Medicine, Jinan University, Guangzhou, China, ${ }^{2}$ College of Pharmacy, Jinan University, Guangzhou, China, ${ }^{3}$ Shenzhen Hospital of Guangzhou University of Chinese Medicine, Shenzhen, China, ${ }^{4}$ Department of Hepatobiliary Surgery, Jinan University First Affiliated Hospital, Guangzhou, China

Angiogenesis is required for tumor growth and development. Extracellular vesicles (EVs) are important signaling entities that mediate communication between diverse types of cells and regulate various cell biological processes, including angiogenesis. Recently, emerging evidence has suggested that tumor-derived EVs play essential roles in tumor progression by regulating angiogenesis. Thousands of molecules are carried by EVs, and the two major types of biomolecules, noncoding RNAs (ncRNAs) and proteins, are transported between cells and regulate physiological and pathological functions in recipient cells. Understanding the regulation of EVs and their cargoes in tumor angiogenesis has become increasingly important. In this review, we summarize the effects of tumor-derived EVs and their cargoes, especially ncRNAs and proteins, on tumor angiogenesis and their mechanisms, and we highlight the clinical implications of EVs in bodily fluids as biomarkers and as diagnostic, prognostic, and therapeutic targets in cancer patients.

Keywords: extracellular vesicles, tumor angiogenesis, miRNAs, IncRNAs, CircRNAs, proteins

\section{INTRODUCTION}

Angiogenesis, defined as the establishment of new blood vessels from pre-existing vascular networks, is triggered by proangiogenic factors and depends on the proliferation and migration of endothelial cells (ECs) (Teleanu et al., 2019; Lugano et al., 2020). In normal healthy tissues, angiogenesis is tightly regulated by a balance that is maintained between proangiogenic and antiangiogenic factors. Solid tumors are generally characterized with aberrant angiogenesis, and tumor angiogenesis is critically required for tumor growth and development (Teleanu et al., 2019; Lugano et al., 2020). Many proangiogenic factors are upregulated in tumor cells and tumor-associated stromal cells, including vascular endothelial growth factor (VEGF), fibroblast growth factor (FGF), and delta ligand-like 4 (Dll4). Hypoxia is a key inducer of tumor angiogenesis and promotes the expression of various proangiogenic factors in the tumor microenvironment (Abou Khouzam et al., 2020). Recently, antiangiogenic drugs have been widely applied to the treatment of multiple solid cancers, and cancer patients have gained tremendous survival benefits from antiangiogenic therapy.

Extracellular vesicles (EVs), such as microvesicles and exosomes, are nanosized vesicles with lipid membranes that are secreted by most cells. EVs contain many bioactive molecules, such as microRNAs (miRNAs), long noncoding RNAs (lncRNAs), circular RNAs (circRNAs), and proteins, and these EV cargoes regulate intercellular communication (Mathieu et al., 2019; Liu 
et al., 2021). Donor cell-derived EVs are taken up by recipient cells, and the encapsulated bioactive components are thus delivered to recipient cells, enabling their regulation of recipient cell biological behaviors. An increasing number of studies have demonstrated that EVs play important roles in tumorigenesis, tumor growth, metastasis, immune evasion, drug resistance, and angiogenesis (Todorova et al., 2017; Aslan et al., 2019). Tumor-derived EVs can transfer proangiogenic molecules into ECs to promote their angiogenic activity via various mechanisms such as VEGF/VEGF Receptor (VEGF/ VEGFR), Notch, Wingless-type (WNT), and Hypoxiainducible factor (HIF) signaling pathway (Phng et al., 2009; Horie et al., 2017; Todorova et al., 2017; Aslan et al., 2019). Thus, targeting EVs might be an innovative and promising therapeutic strategy to inhibit tumor angiogenesis.

A wide variety of biomolecules, including ncRNAs and proteins, have been identified as EV cargoes, and these signaling molecules can be transported from donor cells to recipient cells. To date, considerable attention has been directed to the effects of EVs on tumor angiogenesis and the clinical relevance of these effects. A database of exosomes (http:// www.exocarta.org/) includes 9,769 proteins, 3,408 mRNAs, and 2,838 miRNAs. The mechanisms triggered by these specific cargos loaded into EVs and delivered from donor cells to acceptor cells are complex (Abels and Breakefield, 2016; Mathieu et al., 2019). This article summarizes the current knowledge on the roles of tumor-derived EVs in angiogenesis, with a particular emphasis on the molecular mechanisms involved. We also discuss the main prospects for their applications in cancer diagnosis, prognosis, and treatment.

\section{EXTRACELLULAR VESICLES AND TUMOR ANGIOGENESIS}

\subsection{EV-Derived ncRNAs and Tumor Angiogenesis}

Here, we focus on the effects and mechanisms of EV-derived miRNAs, lncRNAs, and circRNAs on angiogenesis, aiming to elucidate their potential as tumor biomarkers and therapeutic targets for tumor angiogenesis.

\subsection{1 miRNAs}

Various miRNAs are packaged into tumor-derived EVs and can be transferred into recipient ECs (Muralidharan-Chari et al., 2009). Once internalized by ECs, these miRNAs can initiate an angiogenic switch by modulating EC proliferation and migration and regulating the expression of angiogenesis-related genes (Huang et al., 2020a; Li et al., 2020; Masoumi-Dehghi et al., 2020).

VEGF/VEGFR and HIF signaling pathways are the main targets of miRNAs that regulate angiogenesis. Exosomal miR130a secreted by gastric cancer (GC) cells targeted c-MYB in ECs and promoted angiogenesis in vitro and in vivo (Yang et al., 2018). Similarly, GC cell-derived exosomal miR-155 downregulated c-MYB but increased the expression of VEGF in ECs, which enhanced EC tube formation and increased microvessel density in xenografted tumors (Deng et al., 2020). Moreover, inhibition of signal transducer and activator of transcription 3 (STAT3) reduced miR-21 levels in exosomes derived from transformed human bronchial epithelial cells, and these exosomes suppressed angiogenesis by blocking the STAT3/VEGF axis in ECs (Liu et al., 2016). MiR-182-5p in glioblastoma-derived EVs directly targeted Kruppel like factor 2 (KLF2) and KLF4, which resulted in VEGFR accumulation in ECs and thus promoted angiogenesis ( $\mathrm{Li}$ et al., 2020). In addition, HIF is a critical angiogenesis inducer that regulates the cellular response to hypoxia-induced stress (Shao et al., 2018). Under hypoxic conditions, HIF-1a is stabilized and its expression is increased, which facilitates the expression of various proangiogenic factors (Horie et al., 2017). Tumor cellderived exosomal miRNAs, such as miR-21-5p, miR-23a, miR155, miR-181a, miR-182-5p, and miR-619-5p, were also upregulated under hypoxia. Prolyl hydroxylase (PHD) is a negative regulator of HIFs, and inhibition of $\mathrm{PHD}$ can induce the accumulation of HIFs in cells. Exosomal miR-23a derived from hypoxic lung cancer cells inhibited the expression of PHD1 and PHD2 and led to the accumulation of HIF-1 $\alpha$ in ECs, thereby enhancing angiogenesis (Hsu et al., 2017).

EVs derived from tumor stromal cells, such as cancerassociated fibroblasts (CAFs) and tumor-associated macrophages (TAMs), can trigger tumor angiogenesis via various mechanisms. Tumor-derived EVs can promote the transformation of fibroblasts into CAFs and induce M2 polarization of macrophages, thereby inducing the proangiogenic macrophage phenotype switch. For example, lung cancer cell-secreted exosomal miR-210 activated the janus kinase 2 (JAK2)/STAT3 pathway by targeting ten-eleven translocation 2 (TET2) in fibroblasts and thus initiated the acquisition of the proangiogenic phenotype in CAFs, as indicated by the upregulation of VEGFA, MMP9, and FGF2 (Fan et al., 2020). TAMs are immune cells that play a significant role in tumor angiogenesis (Zheng et al., 2018) and M2 macrophages express high levels of proangiogenic factors such as VEGF (Corliss et al., 2016). M2 macrophages were associated with increased microvessel density in pancreatic ductal adenocarcinoma (PDAC) tissues, and exosomal miR-155-5p and miR-211-5p derived from M2 macrophages targeted E2F transcription factor $2(\mathrm{E} 2 \mathrm{~F} 2)$ and promoted the angiogenic functions of mouse aortic ECs in vitro (Yang et al., 2021).

Collectively, miRNA-derived from tumor-secreted EVs regulate angiogenesis primarily by modulating the VEGF/ VEGFR and HIF-1a signaling pathways. In addition to those derived from tumor cells, EVs derived from CAFs and TAMs have been shown to regulate tumor angiogenesis via various mechanisms. The effects and mechanisms of other EV-derived miRNAs on tumor angiogenesis are summarized in Table $\mathbf{1 .}$

\subsubsection{LncRNAs}

Tumor-secreted EV-derived lncRNAs can be transmitted to ECs where they promote the expression of proangiogenic genes and initiate angiogenesis by either binding to endogenous miRNAs or interacting with mRNAs and proteins (Ma et al., 2017; De Los Santos et al., 2019; Zhang et al., 2020). For example, IncRNA-H19 functions as an oncogene and is upregulated in multiple types of cancer (Iempridee, 2017). Exosomes derived from CD90 ${ }^{+}$liver 
TABLE 1 | The effects and mechanisms of miRNAs, IncRNAs, and circRNAs derived from tumor EVs on angiogenesis.

\begin{tabular}{|c|c|c|c|c|c|}
\hline Cargoes & Cancer types & Recipient cells & Target genes or signaling pathways & Functions & References \\
\hline \multicolumn{6}{|l|}{ MiRNAs } \\
\hline \multirow[t]{2}{*}{ miR-9 } & NPC & HUVECs & MDK, PDK/Akt pathway & Inhibition & Lu et al. (2018) \\
\hline & Glioma & HUVECs & COL18A1, THBS2, PTCH1, PHD3, HIF-1a, VEGF & Promotion & Chen et al. (2019) \\
\hline$m i R-17-5 p$ & NPC & HUVECs & $\mathrm{BAMBI}$ & Promotion & Duan et al. (2019) \\
\hline miR-21 & ESCC & HUVECs & SPRY1 & Promotion & Zhuang et al. (2020) \\
\hline $\operatorname{miR}-21-5 p$ & Hypoxic PTC & HUVECS & TGFBI, COL4A1 & Promotion & Wu et al. (2019a) \\
\hline \multirow[t]{3}{*}{ miR-23a } & Hypoxic HCC & HUVECs & SIRT1 & Promotion & Sruthi et al. (2018) \\
\hline & NPC & HUVECs & TSGA10 & Promotion & Bao et al. (2018) \\
\hline & GC & HUVECs & PTEN & Promotion & Du et al. (2020) \\
\hline miR-25-3p & $\mathrm{CRC}$ & HUVECs & KLF2, KLF4, VEGFR2, ZO-1, Occludin, Claudin5 & Promotion & Zeng et al. (2018) \\
\hline miR-26a & Glioma stem cells & HBMECs & PTEN, PI3K/Akt pathway & Promotion & Wang et al. (2019c) \\
\hline \multirow[t]{2}{*}{$\operatorname{miR}-27 a$} & $\mathrm{PC}$ & HMVECs & BTG2 & Promotion & Shang et al. (2020b) \\
\hline & ccRCC & HUVECs & SFRP1 & Promotion & Hou et al. (2021) \\
\hline miR-92a-3p & Retinoblastoma & HUVECs & KLF2 & Promotion & Chen et al. (2021a) \\
\hline $\mathrm{miR}-130 \mathrm{a}$ & GC & HUVECs & c-MYB & Promotion & Yang et al. (2018) \\
\hline miR-130b-3p & OSCC & HUVECs & PTEN & Promotion & Yan et al. (2021) \\
\hline $\operatorname{miR}-135 b$ & $\mathrm{GC}$ & HUVECs & FOXO1 & Promotion & Bai et al. (2019) \\
\hline miR-135b-5p & CAFs from CRC & HUVECs & TXINP & Promotion & Yin et al. (2021) \\
\hline $\operatorname{miR}-141$ & SCLC & HUVECs & KLF12 & Promotion & Mao et al. (2020) \\
\hline $\operatorname{miR}-141-3 p$ & EOC & HUVECs & SOCS5, VEGFR2, JAK/STAT3 and NF-кB signaling pathways & Promotion & $\begin{array}{l}\text { Masoumi-Dehghi } \\
\text { et al. (2020) }\end{array}$ \\
\hline $\operatorname{miR}-148 a-3 p$ & Glioma & HUVECs & ERRFI1, EGFR/MAPK signaling pathway & Promotion & Wang et al. (2020b) \\
\hline $\operatorname{miR}-155$ & $\mathrm{GC}$ & HUVECs & c-MYB/VEGFA axis & Promotion & Deng et al. (2020) \\
\hline \multirow[t]{3}{*}{ miR-155 } & GC & HUVECs & FOXO3a & Promotion & Zhou et al. (2019) \\
\hline & Melanoma & fibroblasts & SOCS1/JAK2/STAT axis, VEGFA, FGF2, MMP9 & Promotion & Zhou et al. (2018) \\
\hline & Hypoxic HCC & HUVECs & - & Promotion & $\begin{array}{l}\text { Matsuura et al. } \\
(2019)\end{array}$ \\
\hline $\operatorname{miR}-155-5 p$ & M2 macrophages & MAECs & Targets E2F2 in PDAC & Promotion & Yang et al. (2021) \\
\hline \multicolumn{6}{|l|}{$\operatorname{miR}-221-5 p$} \\
\hline $\operatorname{miR}-181 a$ & Hypoxic РTC & HUVECs & DACT2, MLL3, YAPNEGF axis & Promotion & Wang et al. (2021b) \\
\hline $\operatorname{miR}-182-5 p$ & Hypoxic GBM & HUVECs & KLF2, KLF4, VEGFR, ZO-1, occludin, claudin-5 & Promotion & Li et al. (2020) \\
\hline $\operatorname{miR}-183-5 p$ & $\mathrm{CRC}$ & HMEC-1 & FOXO1 & Promotion & Shang et al. (2020a) \\
\hline miR-205 & OC & HUVECs & PTEN/Akt pathway & Promotion & He et al. (2019) \\
\hline \multirow[t]{2}{*}{$\operatorname{miR}-210$} & LC & CAFs & JAK2/STAT3 & Promotion & Fan et al. (2020) \\
\hline & $\mathrm{HCC}$ & HUVECs & SMAD4, STAT6 & Promotion & Lin et al. (2018) \\
\hline $\operatorname{miR}-210-3 p$ & OSCC & HUVECs & EFNA3, PI3K/Akt pathway & Promotion & Wang et al. (2020a) \\
\hline \multirow[t]{2}{*}{$\operatorname{miR}-221-3 p$} & CSCC & HUVECs & THBS2 & Promotion & Wu et al. (2019b) \\
\hline & $\mathrm{CC}$ & MVECs & MAPK10 & Promotion & Zhang et al. (2019) \\
\hline miR-378b & $\mathrm{HCC}$ & HUVECs & TGFBR3 & Promotion & Chen et al. (2021b) \\
\hline $\operatorname{miR}-549 a$ & TKI-resistant ccRCC & HUVECs & HIF-1 $1 \alpha$, VEGF & Promotion & Xuan et al. (2021) \\
\hline miR-619-5p & Hypoxic NSCLC & HUVECs & RCAN1.4 & Promotion & Kim et al. (2020) \\
\hline miR-944 & Glioma stem cells & HUVECs & VEGFC, Akt, Erk1/2 signaling pathway & Inhibition & Jiang et al. (2021) \\
\hline miR-1229 & $\mathrm{CRC}$ & HUVECS & HIPK2, VEGF pathway & Promotion & Hu et al. (2019) \\
\hline miR-1260b & NSCLC & HUVECs & HIPK2 & Promotion & Kim et al. (2021) \\
\hline miR-1290 & $\mathrm{HCC}$ & HUVECs & SMEK1 & Promotion & Wang et al. (2021a) \\
\hline miR-3157-3p & NSCLC & HUVECs & TIMP2, KLF2, VEGF, MMP2, MMP9, occludin & Promotion & Ma et al. (2021) \\
\hline $\operatorname{miR}-3682-3 p$ & $\mathrm{HCC}$ & HUVECs & ANGPT1, RAS-MEK1/2-ERK1/2 signaling pathway & Inhibition & Dong et al. (2021) \\
\hline \multicolumn{6}{|l|}{ LncRNAs } \\
\hline LncRNA H19 & Glioma & HBMVECS & miR-29a, VASH2 & Promotion & Jia et al. (2016) \\
\hline LncRNA H19 & $\mathrm{CD}^{+} 0^{+}$liver cancer & HUVECs & VEGF, VEGFR, ICAM1 & Promotion & $\begin{array}{l}\text { Conigliaro et al. } \\
\text { (2015) }\end{array}$ \\
\hline LncRNA HOTAIR & Glioma & HBMVECs & VEGFA & Promotion & Ma et al. (2017) \\
\hline LncRNA CCAT2 & Glioma & HUVECs & VEGFA, TGF $\beta$ & Promotion & Lang et al. (2017b) \\
\hline LncRNA POU3F3 & Glioma & HBMECs & bFGF, FGFR, VEGFA, and ANG & Inhibition & Lang et al. (2017a) \\
\hline LncRNA MALAT1 & EOC & HUVECs & VEGFA, VEGFD, ENA78, PIGF, IL8, ANG, bFGF, Leptin & Promotion & Qiu et al. (2018) \\
\hline LncRNA GAS5 & LC & HUVECs & miR-29-3p, PTEN & Inhibition & Cheng et al. (2019) \\
\hline LncRNA p21 & NSCLC & HUVECs & miR-23a, miR-146b, miR-330, miR-494 & Promotion & $\begin{array}{l}\text { Castellano et al. } \\
(2020)\end{array}$ \\
\hline LncRNA UCA1 & PC & HUVECs & miR-96-5p/AMOTL2/ERK1/2 axis & Promotion & Guo et al. (2020) \\
\hline LncRNA RAMP2-AS1 & Chondrosarcoma & HUVECS & miR-2355-5p/NEGFR axis & Promotion & Cheng et al. (2020) \\
\hline LncRNA APC1 & $\mathrm{CRC}$ & HUVECs & Rab5b, MAPK & Promotion & Wang et al. (2019a) \\
\hline LncRNA TUG1 & $\mathrm{CC}$ & HUVECs & - & $\begin{array}{l}\text { Promotion } \\
\text { (Continuec }\end{array}$ & $\begin{array}{l}\text { Lei and Mou, (2020) } \\
\text { on following page) }\end{array}$ \\
\hline
\end{tabular}


TABLE 1 | (Continued) The effects and mechanisms of miRNAs, IncRNAs, and circRNAs derived from tumor EVs on angiogenesis.

\begin{tabular}{|c|c|c|c|c|c|}
\hline Cargoes & Cancer types & Recipient cells & Target genes or signaling pathways & Functions & References \\
\hline LncRNA X26 nt & GC & HUVECS & VE-cadherin & Promotion & Chen et al. (2021c) \\
\hline LncRNA OIP5-AS1 & Osteosarcoma & HUVECS & miR-153, ATG5 & Promotion & Li et al. (2021c) \\
\hline LncRNA AC073352.1 & $\mathrm{BC}$ & HUVECS & YBX1 stabilization & Promotion & Kong et al. (2021) \\
\hline LncRNA SNHG16 & $\mathrm{HCC}$ & HUVECS & miR-4500/GALNT1 axis, PI3K/Akt/mTOR pathway & Promotion & Li et al. (2021b) \\
\hline LncRNA CCAT1 & $\mathrm{PC}$ & HUVECS & miR-1138-5p/HMGA1 axis & Promotion & Han et al. (2021) \\
\hline LncRNA LINC00161 & $\mathrm{HCC}$ & HUVECS & miR-590-3p/ROCK axis & Promotion & You et al. (2021) \\
\hline LncRNA SNHG11 & $\mathrm{PC}$ & HUVECS & miR-324-3p/NEGFA axis & Promotion & Fang et al. (2021) \\
\hline \multicolumn{6}{|l|}{ CicrRNAs } \\
\hline Circ-100338 & $\mathrm{HCC}$ & HUVECs & MMP9 & Promotion & Huang et al. (2020b) \\
\hline Circ-SHKBP1 & GC & - & miR-582-3p/HUR/NEGF axis & Promotion & Xie et al. (2020b) \\
\hline Circ-RanGAP1 & GC & HUVECS & miR-877-3p/NEGFA axis & Promotion & Lu et al. (2020) \\
\hline Circ-CCAC1 & CCA & HUVECS & SH3GL2, EZH2, ZO-1, Occludin & Promotion & Xu et al. (2021) \\
\hline Circ-0044366 & GC & HUVECs & miR-29aNEGF axis & Promotion & Li et al. (2021a) \\
\hline Circ-CMTM3 & $\mathrm{HCC}$ & HUVECS & miR-3619-5p/SOX9 & Promotion & Hu et al. (2021) \\
\hline
\end{tabular}

Abbreviation: Breast cancer, BC; Cervical cancer, CC, Cervical squamous cell carcinoma; CSCC; Clear cell renal cell carcinoma, cCRCC; Cholangiocarcinoma, CCA; Colorectal cancer, CRC; Epithelial ovarian cancer, EOC; Esophageal squamous cell carcinoma, ESCC; Gastric cancer, Glioblastoma, GBM; GC; Hepatocellular carcinoma, HCC; Lung cancer, LC; Mouse aortic endothelial cells, MAECs; Nasopharyngeal carcinoma, NPC; Non-small cell lung cancer, NSCLC; Ovarian cancer, OC; Oral squamous cell carcinoma, OSCC; Pancreatic cancer, PC; Pancreatic ductal adenocarcinoma, PDAC; Papillary thyroid cancer, PTC; Small cell lung cancer, SCLC., Tyrosine kinase inhibitor, TKI.
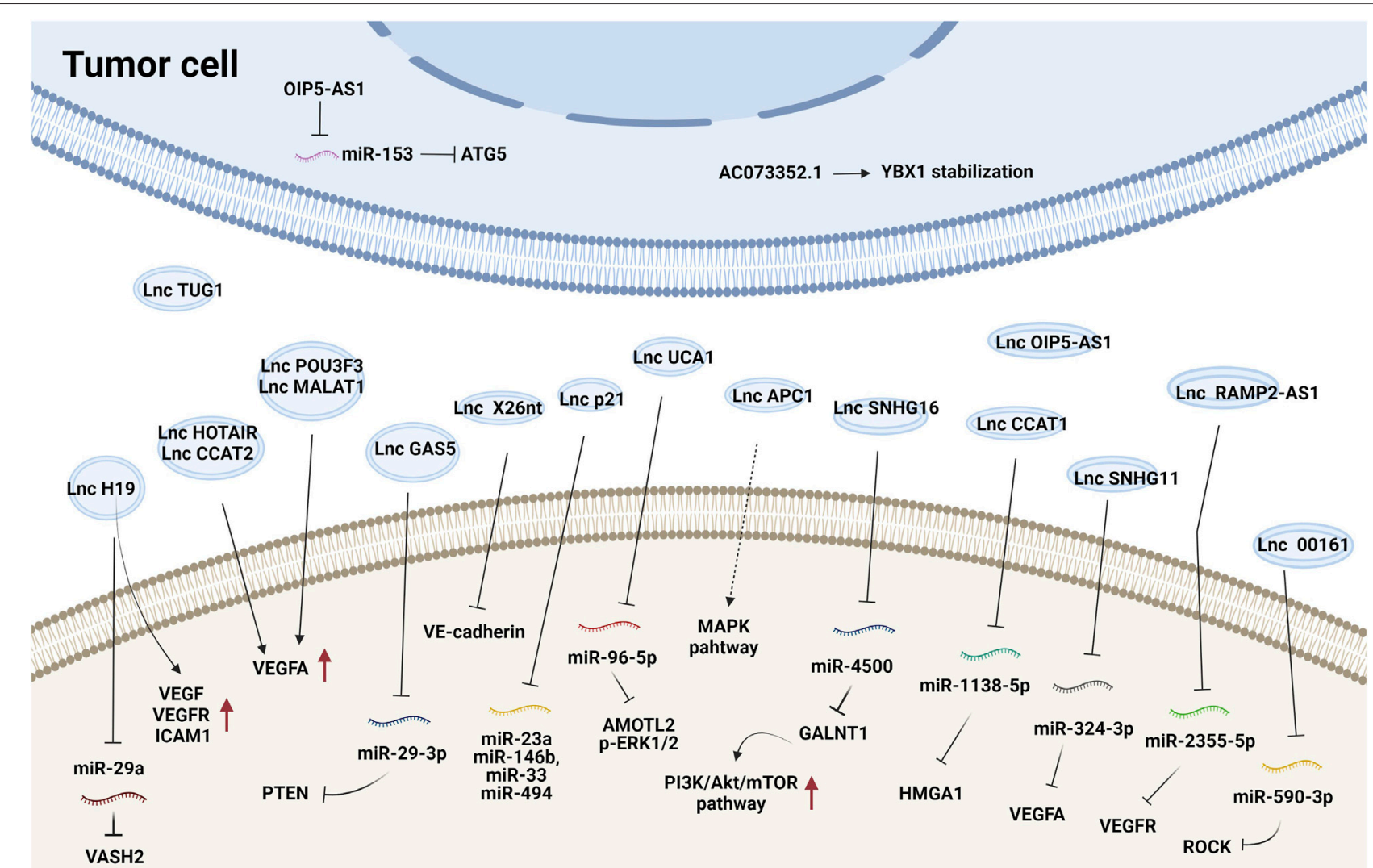

\section{Endothelial cell}

FIGURE 1 | The effects and mechanisms of IncRNAs derived from tumor EVs on angiogenesis. 


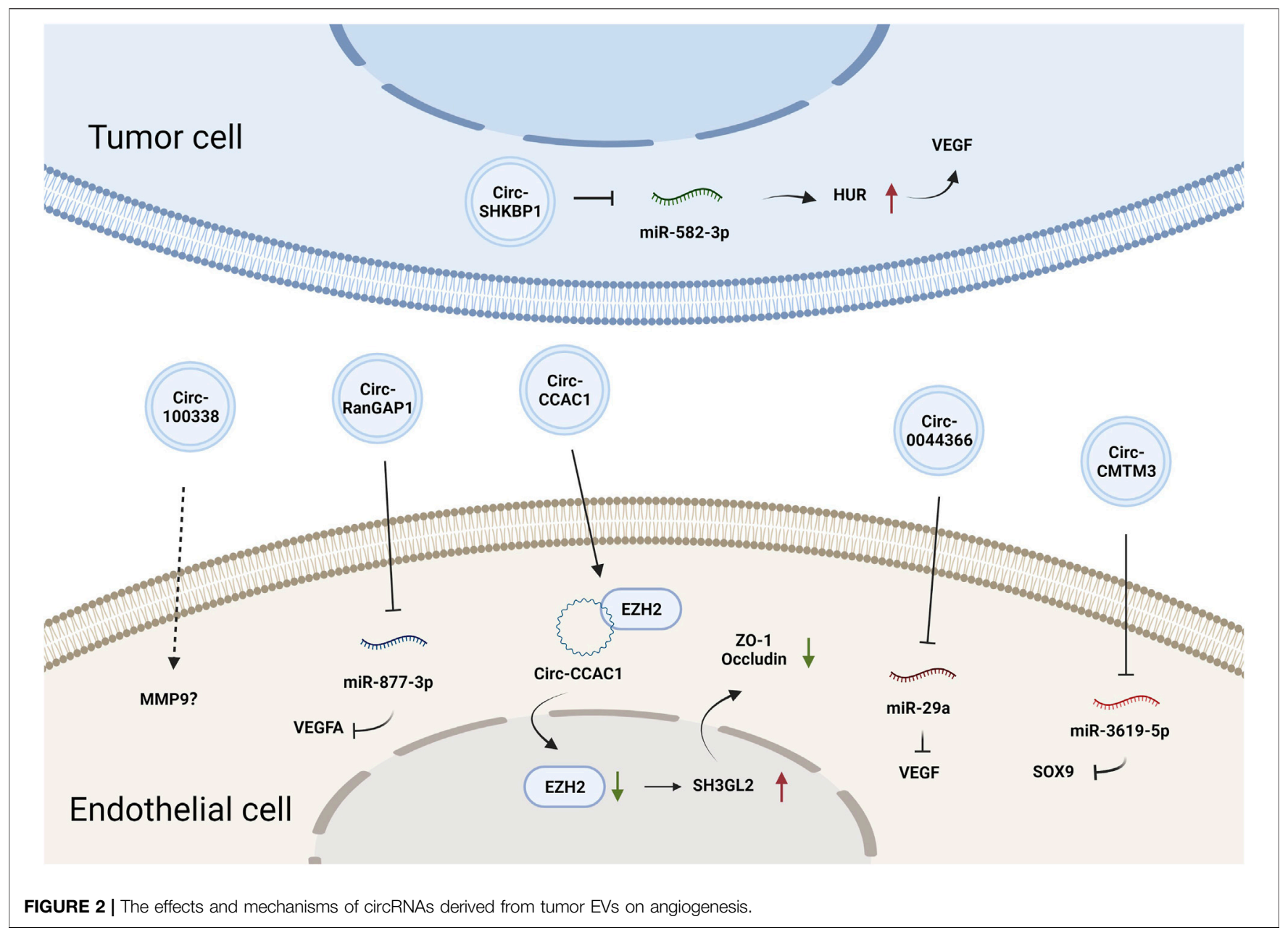

cancer cells were found to be enriched in lncRNA H19 and promoted the angiogenic phenotype of human umbilical vein endothelial cells (HUVECs), probably by regulating VEGF and VEGFR1 expression (Conigliaro et al., 2015). Chondrosarcoma cell-derived exosomes containing IncRNA-RAMP2-AS1 promoted the proliferation, migration and tube formation of ECs by upregulating VEGFR2 by sponging miR-2355-5p (Cheng et al., 2020). LncRNA-UCA1 was highly expressed in exosomes derived from hypoxic pancreatic cancer (PC) cells and promoted angiogenesis and tumor growth by regulating the miR-96-5p/ AMOTL2/ERK1/2 axis (Guo et al., 2020). PC-derived exosomal lncRNA SNHG11 promoted the expression of VEGFA by sponging miR-324-3p (Fang et al., 2021). Additionally, gliomaderived exosomal lncRNA-CCAT2 (Lang et al., 2017b) and lncRNA-POU3F3 (Lang et al., 2017a) enhanced angiogenesis by inducing VEGFA expression. LncRNA-APC1, a suppressor of angiogenesis, was significantly downregulated in colorectal cancer cell-derived EVs. It directly bound to and degraded Rab5b mRNA to decrease EV production and block the mitogenactivated protein kinase (MAPK) signaling pathway in HUVECs to suppress angiogenesis (Wang et al., 2019a). Together, these studies demonstrate that tumor exosomal lncRNAs regulate angiogenesis mainly by modulating VEGFA expression and the VEGF/VEGFR and MAPK pathways. The effects and mechanisms of other EV-derived lncRNAs on tumor angiogenesis are summarized in Figure 1 and Table 1.

\subsubsection{CircRNAs}

CircRNAs constitute a class of endogenous ncRNAs that form a covalently closed loop without a $5^{\prime}$-cap or $3^{\prime}$-poly-A tail (Gan et al., 2021). They are produced by backsplicing protein-coding precursor mRNAs and regarded as variants of competitive endogenous (ceRNAs) that can sponge and thus inhibit the activity of miRNAs (Hansen et al., 2013). Accumulating evidence has demonstrated that circRNAs are involved in various biological processes by regulating gene expression at the transcriptional or posttranscriptional levels ( $\mathrm{Du}$ et al., 2016). CircRNAs can also be loaded into EVs and mediate cell-cell communication. Circ-SHKBP1 in GC cell-derived exosomes promoted angiogenesis by sponging miR-582-3p and thus increased the expression of hu-antigen R (HUR), which regulated VEGF mRNA stability (Xie et al., 2020b). CircRanGAP1 in secreted exosomes derived from the plasma of GC patients and promoted GC progression by targeting the miR-877-3p/VEGFA axis (Lu et al., 2020). Additionally, circ0044366/circ29, which is highly expressed in GC cell-derived 
exosomes, was delivered into ECs and sponged miR-29a to promote angiogenesis by upregulating VEGF (Li et al., 2021a). In summary, tumor EV-derived circRNAs affect tumor angiogenesis primarily by regulating VEGF expression. The effects and mechanisms of other EV-derived circRNAs on tumor angiogenesis are summarized in Figure 2 and Table 1.

\subsection{EV-Derived Proteins and Tumor Angiogenesis}

In recent years, researchers have identified thousands of proteins from different types of tumor-derived EVs, and some of these proteins were characterized with proangiogenic properties and can stimulate various steps in the angiogenic cascade. For example, EVs derived from colorectal cancer perivascular cells contained growth arrest specific 6 (Gas6) and promoted the recruitment of endothelial progenitor cells (EPCs) to tumors by activating the Axl pathway, thus leading to tumor revascularization after withdrawal of antiangiogenic drugs (Huang et al., 2021). VEGFA was carried in EVs derived from ex vivo cultured patient-derived glioblastoma stem-like cells and promoted angiogenesis of human brain ECs (Treps et al., 2017). Breast cancer cell-derived EVs contained $\mathrm{VEGF}_{90 \mathrm{~K}}$, which was generated by $\mathrm{VEGF}_{165}$ crosslinking and triggered sustained activation of VEGFRs in ECs by interacting with heat shock protein 90 (HSP90) (Feng et al., 2017). Furthermore, EVs secreted by ovarian (ES2), colorectal (HCT116), and renal (786-0) cancer cells, in bodily fluids of tumor-bearing mice, and in ovarian cancer patient ascites could stimulate EC migration and tube formation. These responses were mediated by the 189 amino acid isoform of VEGF ( $\mathrm{VEGF}_{189}$ ), which was bound to the surface of these EVs because of its high affinity for heparin (Ko et al., 2019). Collectively, these findings indicate that proangiogenic factors (e.g., Gas6 and VEGFA) and different subtypes of VEGF promote tumor angiogenesis through different mechanisms.

In addition to conventional proangiogenic cytokines, other angiogenesis-related proteins have also been found in EVs. Ephrin type B receptor 2 (EPHB2) in small EVs derived from head and neck squamous cell carcinoma (HNSCC) activated ephrin-B reverse signaling and induced STAT3 phosphorylation in ECs, which promoted angiogenesis both in vitro and in vivo (Sato et al., 2019). Moreover, soluble E-cadherin, which was localized to the surface of exosomes derived from ovarian cancer (OV) cells, activated the $\beta$-catenin and nuclear factor- $\kappa \mathrm{B}(\mathrm{NF}-\kappa \mathrm{B})$ signaling pathways by interacting with VE-cadherin on ECs, leading to angiogenesis in vitro and in vivo (Tang et al., 2018). Exosomal Annexin II secreted by breast cancer cells promoted tPAdependent angiogenesis in vitro and in vivo (Maji et al., 2017). Wnt5A induced the secretion of exosomes containing proangiogenic proteins (e.g., VEGF and MMP2) and immunomodulatory factors (e.g., IL-8 and IL-6) by melanoma cells (Ekstrom et al., 2014). Additionally, other angiogenic proteins have been found in many cancer cell-secreted EVs, such as yes-associated protein (YAP) (Wang et al., 2019b), angiopoietin 2 (ANGPT2) (Xie et al., 2020a), profilin 2 (PFN2) (Cao et al., 2020), Dll4 (Sheldon et al., 2010), ANG,
IL-6, IL-8, tissue inhibitor of metalloproteinases-1 (TIMP-1), TIMP-2, activating transcription factor 2 (ATF2), metastasis associated 1 (MTA1), and Rho associated coiled-coil containing protein kinase 1/2 (ROCK1/2) (Skog et al., 2008; Chan et al., 2015; Yi et al., 2015; Ikeda et al., 2021). More proteins in different types of tumor-derived EVs and their proangiogenic mechanisms are summarized in Figure 3 and Table 2.

\section{EXTRACELLULAR VESICLES AND CLINICAL IMPLICATIONS}

As ncRNAs or proteins loaded in EVs can be distributed in various biofluids, such as blood, urine, tears, saliva, milk, and ascites (Keller et al., 2011), the ability to analyze their cargoes and levels in bodily fluids makes them promising biomarkers for cancer diagnosis and prognosis (Sun and Liu, 2014). Liquid biopsy is a noninvasive method of detecting precise information about the tumor environment/status, which can provide information prior to treatment (Rekker et al., 2014). Through liquid biopsy, numerous proangiogenic contents in EVs have been identified.

Similar to that on circulating free DNA or cell-free DNA and several oncoproteins, such as prostate-specific antigen (PSA) and alpha-fetoprotein (AFP), emerging evidence has suggested that $\mathrm{EV}$-associated ncRNAs and proteins can serve as biomarkers and diagnostic, prognostic, and therapeutic targets in cancer patients.

The levels of serum miR-210 and serum-derived exosomal miR-210 were much higher in HCC patients than in healthy donors. A high level of miR-210 was associated with higher microvessel density in HCC patients (Lin et al., 2018). Increased expression of exosomal circRNA-100338 in the serum of HCC patients was associated with tumor growth and angiogenesis in primary and metastatic HCC. Exosomal circRNA-100338 can serve as a predictor of poor prognosis and lung metastasis in HCC patients following curative hepatectomy (Huang et al., 2020b). Serum exosomal Annexin II promoted angiogenesis, and a high level of serum exosomal Annexin II was associated with tumor grade, poor overall survival (OS), and poor disease-free survival in African-American women with triple-negative breast cancer (Chaudhary et al., 2020). Increased expression of lnc-UCA1 was positively correlated with microvessel density in PC tissues. Exosomal lnc-UCA1 levels were greatly increased in PC patient serum and were associated with tumor size, lymphatic invasion, late tumor node and metastasis stage, and poor OS (Guo et al., 2020). The elevated expression of metastasis associated lung adenocarcinoma transcript 1 (MALAT1) in exosomes derived from epithelial ovarian cancer (EOC) patient serum was significantly correlated with an advanced and metastatic phenotype and served as an independent predictive factor for the OS of EOC patients (Qiu et al., 2018). NSCLC patients with high levels of lncRNA-p21 in EVs derived from tumor-draining pulmonary veins exhibited shorter relapse-free survival and OS (Castellano et al., 2020). The level of circ-CCAC1 in the EVs in the serum of cholangiocarcinoma patients was significantly 


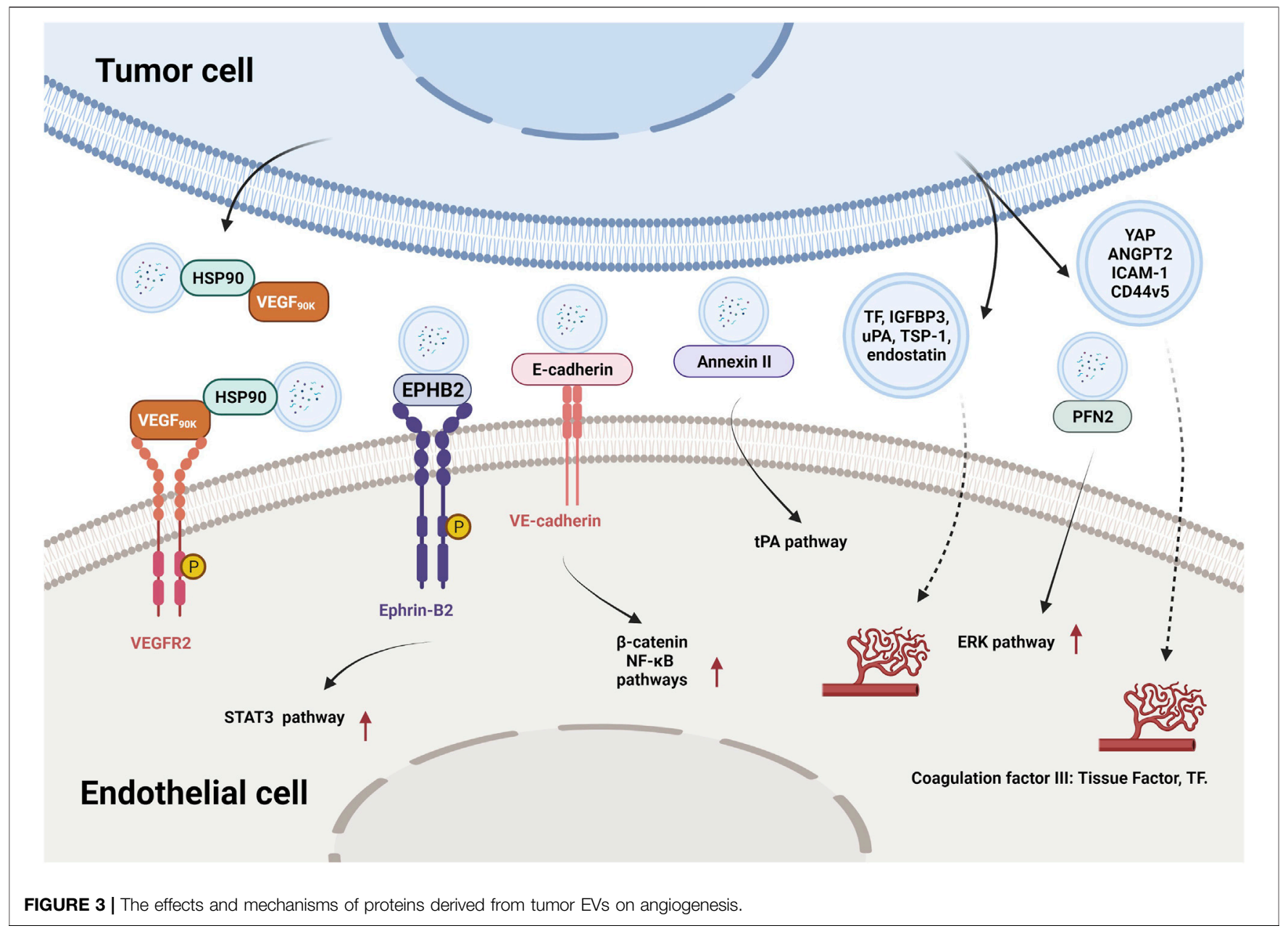

TABLE 2 | The effects and mechanisms of proteins derived from tumor EVs on angiogenesis.

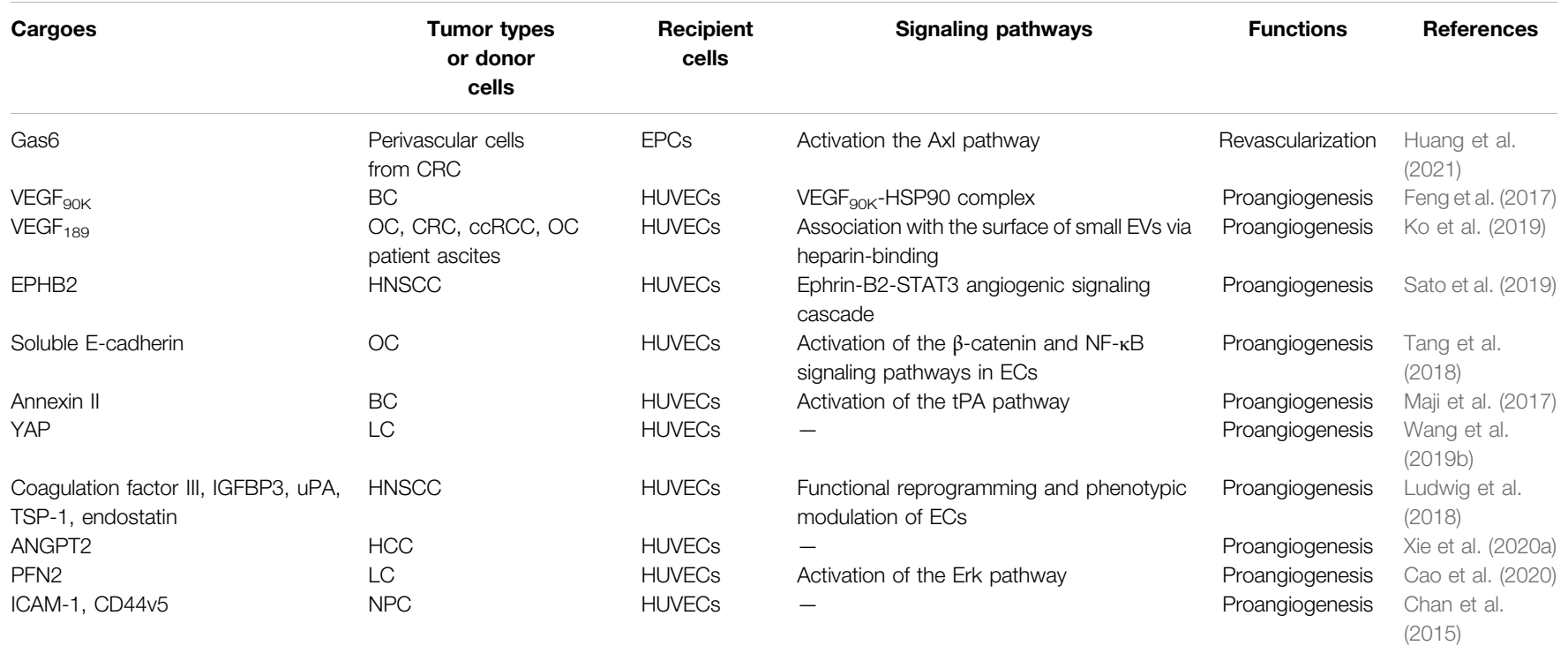

Abbreviations: urokinase type plasminogen activator, UPA; tissue plasminogen activator, tPA. 
increased compared to that of patients with benign hepatobiliary disease, indicating that circ-CCAC1 in EVs may serve as a biomarker for cholangiocarcinoma (Xu et al., 2021). CRC patients with metastasis showed a higher level of miR-25-3p in exosomes than patients without metastasis (Zeng et al., 2018). The expression of miR-619-5p in exosomes was increased in the serum of NSCLC patients, indicating that miR-619-5p can serve as a diagnostic indicator (Kim et al., 2020). High levels of exosomal miR-1260b were associated with high-grade disease, metastasis, and poor survival in patients with NSCLC (Kim et al., 2021).

Moreover, prostate-specific membrane antigen (PSMA) has emerged as a specific prostate tumor biomarker in prostate tumor-derived exosomes. Ziaei et al. developed a novel biofunctionalized silica nanostructure to capture tumorderived exosomes through the interaction of PSMA and its ligand TG97, providing a noninvasive approach for prostate cancer diagnosis (Ziaei et al., 2017). The company MiRXES performed a test to analyze the levels of 12 miRNA biomarkers linked to GC and calculated a cancer risk score for each patient (Kapoor et al., 2020). Another study indicated that the level of phosphatidylserine-expressing tumor-derived exosomes in the blood is a reliable biomarker for early-stage cancer diagnosis (Sharma et al., 2017).

\section{CONCLUSION AND PERSPECTIVES}

Tumor angiogenesis plays a critical role in tumor growth and development, and antiangiogenic therapy has been frequently applied to the clinical treatment of multiple solid tumors. Among the generally known proangiogenic signaling pathways, miRNAs, lncRNAs, circRNAs, and proteins carried by tumor-secreted EVs have recently emerged as important modulators of tumor angiogenesis, acting through a variety of mechanisms, as described in this review.

Antiangiogenic therapy has been widely used for the treatment of various solid tumors and has conferred tremendous survival benefits to cancer patients (Teleanu et al., 2019; Lugano et al., 2020). Antiangiogenic drugs, such as bevacizumab, sorafenib, and regorafenib, inhibit tumor growth by suppressing angiogenesis primarily through blocking the VEGF/VEGFR pathway. However, many patients receive only modest survival benefits and develop acquired resistance to antiangiogenic drugs (Huijbers et al., 2016; Gacche and Assaraf, 2018). Drug resistance is one of the most important obstacles to treatment because it limits the clinical applications of antiangiogenic drugs, and the diseases still progress, which results in poor outcomes and

\section{REFERENCES}

Abels, E. R., and Breakefield, X. O. (2016). Introduction to Extracellular Vesicles: Biogenesis, RNA Cargo Selection, Content, Release, and Uptake. Cell Mol. Neurobiol. 36 (3), 301-312. doi:10.1007/s10571-016-0366-Z

Abou Khouzam, R., Brodaczewska, K., Filipiak, A., Zeinelabdin, N. A., Buart, S., Szczylik, C., et al. (2020). Tumor Hypoxia Regulates Immune Escape/invasion: Influence on unsatisfactory quality of life (Sennino and McDonald, 2012; van Beijnum et al., 2015). Since exosome-derived ncRNAs and proteins play important roles in tumor angiogenesis, targeting ncRNAs and proangiogenic proteins may be a potential therapeutic strategy to inhibit tumor angiogenesis.

Because a single miRNA, lncRNA, and circRNA species has the potential to regulate angiogenesis by modulating multiple targets, these ncRNAs hold great promise for use in therapeutic approaches to the treatment of tumor angiogenesis. However, in addition to tumors, ncRNAs significantly regulate the biological functions of normal cells, and systemic targeting of ncRNAs might affect physiological angiogenesis in normal tissues. Therefore, it is important to develop more specific therapeutic approaches based on angiogenesis-related ncRNAs. Moreover, EVs have turned out to be possible natural carriers of therapeutic agents with long half-time and non-immunogenic properties (Lakhal and Wood, 2011). These EV-based nanocarriers exhibit several advantages such as a high capacity for overcoming various biological barriers and high stability in the blood (Ha et al., 2016). However, the safety, specificity, and proficiency of this promising approach in clinical trials still remain more mysterious. EVs-based nanocarriers still face many challenges in clinical application.

In summary, this review provides deeper insight into the regulatory role of tumor-derived EVs on angiogenesis. Therefore, revealing the mechanisms of tumor-derived EVs on angiogenesis and seeking their potential as biomarkers and diagnostic, prognostic, and therapeutic targets in cancer patients will be popular research directions in the future.

\section{AUTHOR CONTRIBUTIONS}

$\mathrm{LD}, \mathrm{MiH}$ and $\mathrm{MaH}$ designed and revised the manuscript. $\mathrm{MaH}$ and YL drafted the manuscript. YZ, CC, and MW participated in the procedures. All authors listed have made a substantial, direct and intellectual contribution to the work, and approved it for publication.

\section{FUNDING}

This work was supported by the National Natural Science Foundation of China (No. 81803790), National Natural Science Foundation of Guangdong (No. 2020A1515011090) and the Project of Administration of Traditional Chinese Medicine of Guangdong Province of China (Grant no. 20200511205949) to LD.

Angiogenesis and Potential Impact of Hypoxic Biomarkers on Cancer Therapies. Front. Immunol. 11, 613114. doi:10.3389/fimmu.2020.613114

Aslan, C., Maralbashi, S., Salari, F., Kahroba, H., Sigaroodi, F., Kazemi, T., et al. (2019). Tumor-derived Exosomes: Implication in Angiogenesis and Antiangiogenesis Cancer Therapy. J. Cel. Physiol. 129 (2), 727-743. doi:10.1172/JCI122478

Bai, M., Li, J., Yang, H., Zhang, H., Zhou, Z., Deng, T., et al. (2019). miR-135b Delivered by Gastric Tumor Exosomes Inhibits FOXO1 Expression in 
Endothelial Cells and Promotes Angiogenesis. Mol. Ther. 27 (10), 1772-1783. doi:10.1016/j.ymthe.2019.06.018

Bao, L., You, B., Shi, S., Shan, Y., Zhang, Q., Yue, H., et al. (2018). Metastasisassociated miR-23a from Nasopharyngeal Carcinoma-Derived Exosomes Mediates Angiogenesis by Repressing a Novel Target Gene TSGA10. Oncogene 37 (21), 2873-2889. doi:10.1038/s41388-018-0183-6

Cao, Q., Liu, Y., Wu, Y., Hu, C., Sun, L., Wang, J., et al. (2020). Profilin 2 Promotes Growth, Metastasis, and Angiogenesis of Small Cell Lung Cancer through Cancer-Derived Exosomes. Aging 12 (24), 25981-25999. doi:10.18632/ aging. 202213

Castellano, J. J., Marrades, R. M., Molins, L., Viñolas, N., Moises, J., Canals, J., et al. (2020). Extracellular Vesicle lincRNA-P21 Expression in Tumor-Draining Pulmonary Vein Defines Prognosis in NSCLC and Modulates Endothelial Cell Behavior. Cancers 12 (3), 734. doi:10.3390/cancers12030734

Chan, Y.-K., Zhang, H., Liu, P., Tsao, S.-W., Lung, M. L., Mak, N.-K., et al. (2015). Proteomic Analysis of Exosomes from Nasopharyngeal Carcinoma Cell Identifies Intercellular Transfer of Angiogenic Proteins. Int. J. Cancer 137 (8), 1830-1841. doi:10.1002/ijc.29562

Chaudhary, P., Gibbs, L. D., Maji, S., Lewis, C. M., Suzuki, S., and Vishwanatha, J. K. (2020). Serum Exosomal-Annexin A2 Is Associated with AfricanAmerican Triple-Negative Breast Cancer and Promotes Angiogenesis. Breast Cancer Res. 22 (1), 11. doi:10.1186/s13058-020-1251-8

Chen, X., Yang, F., Zhang, T., Wang, W., Xi, W., Li, Y., et al. (2019). MiR-9 Promotes Tumorigenesis and Angiogenesis and Is Activated by MYC and OCT4 in Human Glioma. J. Exp. Clin. Cancer Res. 38 (1), 99. doi:10.1186/ s13046-019-1078-2

Chen, S., Chen, X., Luo, Q., Liu, X., Wang, X., Cui, Z., et al. (2021a). Retinoblastoma Cell-Derived Exosomes Promote Angiogenesis of Human Vesicle Endothelial Cells through microRNA-92a-3p. Cell Death Dis 12 (7), 695. doi:10.1038/ s41419-021-03986-0

Chen, W., Huang, L., Liang, J., Ye, Y., He, S., and Niu, J. (2021b). Hepatocellular Carcinoma Cells-Derived Exosomal microRNA-378b Enhances Hepatocellular Carcinoma Angiogenesis. Life Sci. 273, 119184. doi:10.1016/j.lfs.2021.119184

Chen, X., Zhang, S., Du, K., Zheng, N., Liu, Y., Chen, H., et al. (2021c). Gastric Cancer-Secreted Exosomal X26nt Increases Angiogenesis and Vascular Permeability by Targeting VE-cadherin. Cancer Sci. 112 (5), 1839-1852. doi:10.1111/cas.14740

Cheng, Y., Dai, X., Yang, T., Zhang, N., Liu, Z., and Jiang, Y. (2019). Low Long Noncoding RNA Growth Arrest-specific Transcript 5 Expression in the Exosomes of Lung Cancer Cells Promotes Tumor Angiogenesis. J. Oncol. 2019, 1-13. doi:10.1155/2019/2476175

Cheng, C., Zhang, Z., Cheng, F., and Shao, Z. (2020). Exosomal lncRNA RAMP2AS1 Derived from Chondrosarcoma Cells Promotes Angiogenesis through miR-2355-5p/VEGFR2 Axis. Onco Targets Ther. 13, 3291-3301. doi:10.2147/ OTT.S244652

Conigliaro, A., Costa, V., Lo Dico, A., Saieva, L., Buccheri, S., Dieli, F., et al. (2015). CD90+ Liver Cancer Cells Modulate Endothelial Cell Phenotype through the Release of Exosomes Containing H19 IncRNA. Mol. Cancer 14, 155. doi:10.1186/s12943-015-0426-x

Corliss, B. A., Azimi, M. S., Munson, J. M., Peirce, S. M., and Murfee, W. L. (2016). Macrophages: an Inflammatory Link between Angiogenesis and Lymphangiogenesis. Microcirculation 23 (2), 95-121. doi:10.1111/micc.12259

De los Santos, M. C., Dragomir, M. P., and Calin, G. A. (2019). The Role of Exosomal Long Non-coding RNAs in Cancer Drug Resistance. Cdr 2, 1178-1192. doi: $10.20517 / \mathrm{cdr} .2019 .74$

Deng, T., Zhang, H., Yang, H., Wang, H., Bai, M., Sun, W., et al. (2020). Exosome miR-155 Derived from Gastric Carcinoma Promotes Angiogenesis by Targeting the C-MYB/VEGF axis of Endothelial Cells. Mol. Ther. - Nucleic Acids 19, 1449-1459. doi:10.1016/j.omtn.2020.01.024

Dong, S.-S., Dong, D.-D., Yang, Z.-F., Zhu, G.-Q., Gao, D.-M., Chen, J., et al. (2021). Exosomal miR-3682-3p Suppresses Angiogenesis by Targeting ANGPT1 via the RAS-MEK1/2-ERK1/2 Pathway in Hepatocellular Carcinoma. Front. Cel. Dev. Biol. 9, 633358. doi:10.3389/fcell.2021.633358

Du, W. W., Yang, W., Liu, E., Yang, Z., Dhaliwal, P., and Yang, B. B. (2016). Foxo3 Circular RNA Retards Cell Cycle Progression via Forming Ternary Complexes with P21 and CDK2. Nucleic Acids Res. 44 (6), 2846-2858. doi:10.1093/nar/ gkw027
Du, J., Liang, Y., Li, J., Zhao, J.-M., Wang, Z.-N., and Lin, X.-Y. (2020). Gastric Cancer Cell-Derived Exosomal microRNA-23a Promotes Angiogenesis by Targeting PTEN. Front. Oncol. 10, 326. doi:10.3389/fonc.2020.00326

Duan, B., Shi, S., Yue, H., You, B., Shan, Y., Zhu, Z., et al. (2019). Exosomal miR-17$5 p$ Promotes Angiogenesis in Nasopharyngeal Carcinoma via Targeting BAMBI. J. Cancer 10 (26), 6681-6692. doi:10.7150/jca.30757

Ekström, E. J., Bergenfelz, C., von Bülow, V., Serifler, F., Carlemalm, E., Jönsson, G., et al. (2014). WNT5A Induces Release of Exosomes Containing Pro-angiogenic and Immunosuppressive Factors from Malignant Melanoma Cells. Mol. Cancer 13, 88. doi:10.1186/1476-4598-13-88

Fan, J., Xu, G., Chang, Z., Zhu, L., and Yao, J. (2020). miR-210 Transferred by Lung Cancer Cell-Derived Exosomes May Act as Proangiogenic Factor in CancerAssociated Fibroblasts by Modulating JAK2/STAT3 Pathway. Clin. Sci. (Lond) 134 (7), 807-825. doi:10.1042/CS20200039

Fang, X., Cai, Y., Xu, Y., and Zhang, H. (2021). Exosome-Mediated lncRNA SNHG11 Regulates Angiogenesis in Pancreatic Carcinoma through miR-3243p/VEGFA axis. Cell Biol. Int. doi:10.1002/cbin.11703

Feng, Q., Zhang, C., Lum, D., Druso, J. E., Blank, B., Wilson, K. F., et al. (2017). A Class of Extracellular Vesicles from Breast Cancer Cells Activates VEGF Receptors and Tumour Angiogenesis. Nat. Commun. 8, 14450. doi:10.1038/ ncomms 14450

Gacche, R. N., and Assaraf, Y. G. (2018). Redundant Angiogenic Signaling and Tumor Drug Resistance. Drug Resist. Updates 36, 47-76. doi:10.1016/ j.drup.2018.01.002

Gan, H., Lei, Y., Yuan, N., Tang, K., Hao, W., Ma, Q., et al. (2021). Circular RNAs in Depression: Biogenesis, Function, Expression, and Therapeutic Potential. Biomed. Pharmacother. 137, 111244. doi:10.1016/j.biopha.2021.111244

Guo, Z., Wang, X., Yang, Y., Chen, W., Zhang, K., Teng, B., et al. (2020). Hypoxic Tumor-Derived Exosomal Long Noncoding RNA UCA1 Promotes Angiogenesis via miR-96-5p/AMOTL2 in Pancreatic Cancer. Mol. Ther. Nucleic Acids 22, 179-195. doi:10.1016/j.omtn.2020.08.021

Ha, D., Yang, N., and Nadithe, V. (2016). Exosomes as Therapeutic Drug Carriers and Delivery Vehicles across Biological Membranes: Current Perspectives and Future Challenges. Acta Pharm. Sin. B 6 (4), 287-296. doi:10.1016/ j.apsb.2016.02.001

Han, W., Sulidankazha, Q., Nie, X., Yilidan, R., and Len, K. (2021). Pancreatic Cancer Cells-Derived Exosomal Long Non-coding RNA CCAT1/microRNA138-5p/HMGA1 axis Promotes Tumor Angiogenesis. Life Sci. 278, 119495. doi:10.1016/j.lfs.2021.119495

Hansen, T. B., Jensen, T. I., Clausen, B. H., Bramsen, J. B., Finsen, B., Damgaard, C. K., et al. (2013). Natural RNA Circles Function as Efficient microRNA Sponges. Nature 495 (7441), 384-388. doi:10.1038/nature1 1993

He, L., Zhu, W., Chen, Q., Yuan, Y., Wang, Y., Wang, J., et al. (2019). Ovarian Cancer Cell-Secreted Exosomal miR-205 Promotes Metastasis by Inducing Angiogenesis. Theranostics 9 (26), 8206-8220. doi:10.7150/thno.37455

Horie, K., Kawakami, K., Fujita, Y., Sugaya, M., Kameyama, K., Mizutani, K., et al. (2017). Exosomes Expressing Carbonic Anhydrase 9 Promote Angiogenesis. Biochem. Biophys. Res. Commun. 492 (3), 356-361. doi:10.1016/j.bbrc.2017.08.107

Hou, Y., Fan, L., and Li, H. (2021). Oncogenic miR-27a Delivered by Exosomes Binds to SFRP1 and Promotes Angiogenesis in Renal clear Cell Carcinoma. Mol. Ther. - Nucleic Acids 24, 92-103. doi:10.1016/j.omtn.2020.11.019

Hsu, Y.-L., Hung, J.-Y., Chang, W.-A., Lin, Y.-S., Pan, Y.-C., Tsai, P.-H., et al. (2017). Hypoxic Lung Cancer-Secreted Exosomal miR-23a Increased Angiogenesis and Vascular Permeability by Targeting Prolyl Hydroxylase and Tight junction Protein ZO-1. Oncogene 36 (34), 4929-4942. doi:10.1038/onc.2017.105

Hu, H.-Y., Yu, C.-H., Zhang, H.-H., Zhang, S.-Z., Yu, W.-Y., Yang, Y., et al. (2019). Exosomal miR-1229 Derived from Colorectal Cancer Cells Promotes Angiogenesis by Targeting HIPK2. Int. J. Biol. Macromol. 132, 470-477. doi:10.1016/j.ijbiomac.2019.03.221

Hu, K., Li, N. F., Li, J. R., Chen, Z. G., Wang, J. H., and Sheng, L. Q. (2021). Exosome circCMTM3 Promotes Angiogenesis and Tumorigenesis of Hepatocellular Carcinoma through miR-3619-5p/SOX9. Hepatol. Res. 51, 1139-1152. doi:10.1111/hepr.13692

Huang, W., Yan, Y., Liu, Y., Lin, M., Ma, J., Zhang, W., et al. (2020a). Exosomes with Low miR-34c-3p Expression Promote Invasion and Migration of Nonsmall Cell Lung Cancer by Upregulating Integrin $\alpha 2 \beta 1$. Sig Transduct Target. Ther. 5 (1), 39. doi:10.1038/s41392-020-0133-y 
Huang, X.-Y., Huang, Z.-L., Huang, J., Xu, B., Huang, X.-Y., Xu, Y.-H., et al. (2020b). Exosomal circRNA-100338 Promotes Hepatocellular Carcinoma Metastasis via Enhancing Invasiveness and Angiogenesis. J. Exp. Clin. Cancer Res. 39 (1), 20. doi:10.1186/s13046-020-1529-9

Huang, M., Chen, M., Qi, M., Ye, G., Pan, J., Shi, C., et al. (2021). Perivascular Cellderived Extracellular Vesicles Stimulate Colorectal Cancer Revascularization after Withdrawal of Antiangiogenic Drugs. J. Extracell. Vesicles 10 (7), e12096. doi:10.1002/jev2.12096

Huijbers, E. J. M., van Beijnum, J. R., Thijssen, V. L., Sabrkhany, S., NowakSliwinska, P., and Griffioen, A. W. (2016). Role of the Tumor Stroma in Resistance to Anti-angiogenic Therapy. Drug Resist. Updates 25, 26-37. doi:10.1016/j.drup.2016.02.002

Iempridee, T. (2017). Long Non-coding RNA H19 Enhances Cell Proliferation and anchorage-Independent Growth of Cervical Cancer Cell Lines. Exp. Biol. Med. (Maywood) 242 (2), 184-193. doi:10.1177/1535370216670542

Ikeda, A., Nagayama, S., Sumazaki, M., Konishi, M., Fujii, R., Saichi, N., et al. (2021). Colorectal Cancer-Derived CAT1-Positive Extracellular Vesicles Alter Nitric Oxide Metabolism in Endothelial Cells and Promote Angiogenesis. Mol. Cancer Res. 19(5), 834-846. doi:10.1158/15417786.MCR-20-0827

Jia, P., Cai, H., Liu, X., Chen, J., Ma, J., Wang, P., et al. (2016). Long Non-coding RNA H19 Regulates Glioma Angiogenesis and the Biological Behavior of Glioma-Associated Endothelial Cells by Inhibiting microRNA-29a. Cancer Lett. 381 (2), 359-369. doi:10.1016/j.canlet.2016.08.009

Jiang, J., Lu, J., Wang, X., Sun, B., Liu, X., Ding, Y., et al. (2021). Glioma Stem CellDerived Exosomal miR-944 Reduces Glioma Growth and Angiogenesis by Inhibiting AKT/ERK Signaling. Aging 13 (15), 19243-19259. doi:10.18632/ aging.203243

Kapoor, R., So, J. B. Y., Zhu, F., Too, H.-P., Yeoh, K.-G., and Yoong, J. S.-Y. (2020). Evaluating the Use of microRNA Blood Tests for Gastric Cancer Screening in a Stratified Population-Level Screening Program: an Early Model-Based CostEffectiveness Analysis. Value in Health 23 (9), 1171-1179. doi:10.1016/ j.jval.2020.04.1829

Keller, S., Ridinger, J., Rupp, A.-K., Janssen, J. W., and Altevogt, P. (2011). Body Fluid Derived Exosomes as a Novel Template for Clinical Diagnostics. J. Transl. Med. 9, 86. doi:10.1186/1479-5876-9-86

Kim, D. H., Park, S., Kim, H., Choi, Y. J., Kim, S. Y., Sung, K. J., et al. (2020). Tumor-derived Exosomal miR-619-5p Promotes Tumor Angiogenesis and Metastasis through the Inhibition of RCAN1.4. Cancer Lett. 475, 2-13. doi:10.1016/j.canlet.2020.01.023

Kim, D. H., Park, H., Choi, Y. J., Kang, M.-H., Kim, T.-K., Pack, C.-G., et al. (2021). Exosomal miR-1260b Derived from Non-small Cell Lung Cancer Promotes Tumor Metastasis through the Inhibition of HIPK2. Cel Death Dis 12 (8), 747. doi:10.1038/s41419-021-04024-9

Ko, S. Y., Lee, W., Kenny, H. A., Dang, L. H., Ellis, L. M., Jonasch, E., et al. (2019). Cancer-derived Small Extracellular Vesicles Promote Angiogenesis by HeparinBound, Bevacizumab-Insensitive VEGF, Independent of Vesicle Uptake. Commun. Biol. 2, 386. doi:10.1038/s42003-019-0609-X

Kong, X., Li, J., Li, Y., Duan, W., Qi, Q., Wang, T., et al. (2021). A Novel Long Noncoding RNA AC073352.1 Promotes Metastasis and Angiogenesis via Interacting with YBX1 in Breast Cancer. Cel Death Dis 12 (7), 670. doi:10.1038/s41419-021-03943-X

Lakhal, S., and Wood, M. J. A. (2011). Exosome Nanotechnology: An Emerging Paradigm Shift in Drug Delivery: Exploitation of Exosome Nanovesicles for Systemic in Vivo Delivery of RNAi Heralds New Horizons for Drug Delivery Across Biological Barriers. Bioessays 33 (10), 737-741. doi:10.1002/ bies. 201100076

Lang, H. L., Hu, G. W., Chen, Y., Liu, Y., Tu, W., Lu, Y. M., et al. (2017a). Glioma Cells Promote Angiogenesis through the Release of Exosomes Containing Long Non-coding RNA POU3F3. Eur. Rev. Med. Pharmacol. Sci. 21 (5), 959-972.

Lang, H.-L., Hu, G.-W., Zhang, B., Kuang, W., Chen, Y., Wu, L., et al. (2017b). Glioma Cells Enhance Angiogenesis and Inhibit Endothelial Cell Apoptosis through the Release of Exosomes that Contain Long Non-coding RNA CCAT2. Oncol. Rep. 38 (2), 785-798. doi:10.3892/or.2017.5742

Lei, L., and Mou, Q. (2020). Exosomal Taurine Up-Regulated 1 Promotes Angiogenesis and Endothelial Cell Proliferation in Cervical Cancer. Cancer Biol. Ther. 21 (8), 717-725. doi:10.1080/15384047.2020.1764318
Li, J., Yuan, H., Xu, H., Zhao, H., and Xiong, N. (2020). Hypoxic Cancer-Secreted Exosomal miR-182-5p Promotes Glioblastoma Angiogenesis by Targeting Kruppel-like Factor 2 and 4. Mol. Cancer Res. 18 (8), 1218-1231. doi:10.1158/1541-7786.MCR-19-0725

Li, S., Li, J., Zhang, H., Zhang, Y., Wang, X., Yang, H., et al. (2021a). Gastric Cancer Derived Exosomes Mediate the Delivery of circRNA to Promote Angiogenesis by Targeting miR-29a/VEGF axis in Endothelial Cells. Biochem. Biophys. Res. Commun. 560, 37-44. doi:10.1016/j.bbrc.2021.04.099

Li, S., Qi, Y., Huang, Y., Guo, Y., Huang, T., and Jia, L. (2021b). Exosome-derived SNHG16 Sponging miR-4500 Activates HUVEC Angiogenesis by Targeting GALNT1 via PI3K/Akt/mTOR Pathway in Hepatocellular Carcinoma. J. Physiol. Biochem. 77, 667-682. doi:10.1007/s13105-021-00833-w

Li, Y., Lin, S., Xie, X., Zhu, H., Fan, T., and Wang, S. (2021c). Highly Enriched Exosomal lncRNA OIP5-AS1 Regulates Osteosarcoma Tumor Angiogenesis and Autophagy through miR-153 and ATG5. Am. J. Transl. Res. 13 (5), 4211-4223.

Lin, X.-J., Fang, J.-H., Yang, X.-J., Zhang, C., Yuan, Y., Zheng, L., et al. (2018). Hepatocellular Carcinoma Cell-Secreted Exosomal microRNA-210 Promotes Angiogenesis In Vitro and In Vivo. Mol. Ther. - Nucleic Acids 11, 243-252. doi:10.1016/j.omtn.2018.02.014

Liu, Y., Luo, F., Wang, B., Li, H., Xu, Y., Liu, X., et al. (2016). STAT3-regulated Exosomal miR-21 Promotes Angiogenesis and Is Involved in Neoplastic Processes of Transformed Human Bronchial Epithelial Cells. Cancer Lett. 370 (1), 125-135. doi:10.1016/j.canlet.2015.10.011

Liu, J., Ren, L., Li, S., Li, W., Zheng, X., Yang, Y., et al. (2021). The Biology, Function, and Applications of Exosomes in Cancer. Acta Pharmaceutica Sinica B 11, 2783-2797. doi:10.1016/j.apsb.2021.01.001

Lu, J., Liu, Q.-H., Wang, F., Tan, J.-J., Deng, Y.-Q., Peng, X.-H., et al. (2018). Exosomal miR-9 Inhibits Angiogenesis by Targeting MDK and Regulating PDK/AKT Pathway in Nasopharyngeal Carcinoma. J. Exp. Clin. Cancer Res. 37 (1), 147. doi:10.1186/s13046-018-0814-3

Lu, J., Wang, Y.-h., Yoon, C., Huang, X.-y., Xu, Y., Xie, J.-w., et al. (2020). Circular RNA Circ-RanGAP1 Regulates VEGFA Expression by Targeting miR-877-3p to Facilitate Gastric Cancer Invasion and Metastasis. Cancer Lett. 471, 38-48. doi:10.1016/j.canlet.2019.11.038

Ludwig, N., Yerneni, S. S., Razzo, B. M., and Whiteside, T. L. (2018). Exosomes from HNSCC Promote Angiogenesis through Reprogramming of Endothelial Cells. Mol. Cancer Res. 16 (11), 1798-1808. doi:10.1158/1541-7786.MCR-180358

Lugano, R., Ramachandran, M., and Dimberg, A. (2020). Tumor Angiogenesis: Causes, Consequences, Challenges and Opportunities. Cell. Mol. Life Sci. 77 (9), 1745-1770. doi:10.1007/s00018-019-03351-7

Ma, X., Li, Z., Li, T., Zhu, L., Li, Z., and Tian, N. (2017). Long Non-Coding RNA HOTAIR Enhances Angiogenesis by Induction of VEGFA Expression in Glioma Cells and Transmission to Endothelial Cells via Glioma Cell Derived-Extracellular Vesicles. Am. J. Transl. Res. 9 (11), 5012-5021.

Ma, Z., Wei, K., Yang, F., Guo, Z., Pan, C., He, Y., et al. (2021). Tumor-Derived Exosomal miR-3157-3p Promotes Angiogenesis, Vascular Permeability and Metastasis by Targeting TIMP/KLF2 in Non-small Cell Lung Cancer. Cel Death Dis 12 (9), 840. doi:10.1038/s41419-021-04037-4

Maji, S., Chaudhary, P., Akopova, I., Nguyen, P. M., Hare, R. J., Gryczynski, I., et al. (2017). Exosomal Annexin II Promotes Angiogenesis and Breast Cancer Metastasis. Mol. Cancer Res. 15 (1), 93-105. doi:10.1158/1541-7786.Mcr-160163

Mao, S., Lu, Z., Zheng, S., Zhang, H., Zhang, G., Wang, F., et al. (2020). Exosomal miR-141 Promotes Tumor Angiogenesis via KLF12 in Small Cell Lung Cancer. J. Exp. Clin. Cancer Res. 39 (1), 193. doi:10.1186/s13046-020-01680-1

Masoumi-Dehghi, S., Babashah, S., and Sadeghizadeh, M. (2020). microRNA-1413p-containing Small Extracellular Vesicles Derived from Epithelial Ovarian Cancer Cells Promote Endothelial Cell Angiogenesis through Activating the JAK/STAT3 and NF-кB Signaling Pathways. J. Cel Commun. Signal. 14 (2), 233-244. doi:10.1007/s12079-020-00548-5

Mathieu, M., Martin-Jaular, L., Lavieu, G., and Théry, C. (2019). Specificities of Secretion and Uptake of Exosomes and Other Extracellular Vesicles for CellTo-Cell Communication. Nat. Cel Biol. 21 (1), 9-17. doi:10.1038/s41556-0180250-9

Matsuura, Y., Wada, H., Eguchi, H., Gotoh, K., Kobayashi, S., Kinoshita, M., et al. (2019). Exosomal miR-155 Derived from Hepatocellular Carcinoma Cells 
under Hypoxia Promotes Angiogenesis in Endothelial Cells. Dig. Dis. Sci. 64 (3), 792-802. doi:10.1007/s10620-018-5380-1

Muralidharan-Chari, V., Clancy, J., Plou, C., Romao, M., Chavrier, P., Raposo, G., et al. (2009). ARF6-regulated Shedding of Tumor Cell-Derived Plasma Membrane Microvesicles. Curr. Biol. 19 (22), 1875-1885. doi:10.1016/ j.cub.2009.09.059

Phng, L.-K., Potente, M., Leslie, J. D., Babbage, J., Nyqvist, D., Lobov, I., et al. (2009). Nrarp Coordinates Endothelial Notch and Wnt Signaling to Control Vessel Density in Angiogenesis. Dev. Cel 16 (1), 70-82. doi:10.1016/ j.devcel.2008.12.009

Qiu, J.-J., Lin, X.-J., Tang, X.-Y., Zheng, T.-T., Lin, Y.-Y., and Hua, K.-Q. (2018). Exosomal Metastasis-Associated Lung Adenocarcinoma Transcript 1 Promotes Angiogenesis and Predicts Poor Prognosis in Epithelial Ovarian Cancer. Int. J. Biol. Sci. 14 (14), 1960-1973. doi:10.7150/ijbs.28048

Rekker, K., Saare, M., Roost, A. M., Kubo, A.-L., Zarovni, N., Chiesi, A., et al. (2014). Comparison of Serum Exosome Isolation Methods for microRNA Profiling. Clin. Biochem. 47 (1-2), 135-138. doi:10.1016/ j.clinbiochem.2013.10.020

Sato, S., Vasaikar, S., Eskaros, A., Kim, Y., Lewis, J. S., Zhang, B., et al. (2019). EPHB2 Carried on Small Extracellular Vesicles Induces Tumor Angiogenesis via Activation of Ephrin Reverse Signaling. JCI Insight 4 (23), e132447. doi:10.1172/jci.insight.132447

Sennino, B., and McDonald, D. M. (2012). Controlling Escape from Angiogenesis Inhibitors. Nat. Rev. Cancer 12 (10), 699-709. doi:10.1038/nrc3366

Shang, A., Wang, X., Gu, C., Liu, W., Sun, J., Zeng, B., et al. (2020a). Exosomal miR183-5p Promotes Angiogenesis in Colorectal Cancer by Regulation of FOXO1. Aging 12 (9), 8352-8371. doi:10.18632/aging.103145

Shang, D., Xie, C., Hu, J., Tan, J., Yuan, Y., Liu, Z., et al. (2020b). Pancreatic Cancer Cell-Derived Exosomal microRNA-27a Promotes Angiogenesis of Human Microvascular Endothelial Cells in Pancreatic Cancer via BTG2. J. Cel. Mol. Med. 24 (1), 588-604. doi:10.1111/jcmm.14766

Shao, C., Yang, F., Miao, S., Liu, W., Wang, C., Shu, Y., et al. (2018). Role of Hypoxia-Induced Exosomes in Tumor Biology. Mol. Cancer 17 (1), 120. doi:10.1186/s12943-018-0869-y

Sharma, R., Huang, X., Brekken, R. A., and Schroit, A. J. (2017). Detection of Phosphatidylserine-Positive Exosomes for the Diagnosis of Early-Stage Malignancies. Br. J. Cancer 117 (4), 545-552. doi:10.1038/bjc.2017.183

Sheldon, H., Heikamp, E., Turley, H., Dragovic, R., Thomas, P., Oon, C. E., et al. (2010). New Mechanism for Notch Signaling to Endothelium at a Distance by Delta-like 4 Incorporation into Exosomes. Blood 116 (13), 2385-2394. doi:10.1182/blood-2009-08-239228

Skog, J., Wurdinger, T., Van Rijn, S., Meijer, D. H., Gainche, L., Sena-Esteves, M., et al. (2008). Glioblastoma Microvesicles Transport RNA and Proteins That Promote Tumour Growth and Provide Diagnostic Biomarkers. Nat. Cell Biol. 10(12), 1470-1476. doi:10.1038/ncb1800

Sruthi, T. V., Edatt, L., Raji, G. R., Kunhiraman, H., Shankar, S. S., Shankar, V., et al. (2018). Horizontal Transfer of miR-23a from Hypoxic Tumor Cell Colonies Can Induce Angiogenesis. J. Cel. Physiol. 233 (4), 3498-3514. doi:10.1002/ jcp. 26202

Sun, Y., and Liu, J. (2014). Potential of Cancer Cell-Derived Exosomes in Clinical Application: a Review of Recent Research Advances. Clin. Ther. 36 (6), 863-872. doi:10.1016/j.clinthera.2014.04.018

Tang, M. K. S., Yue, P. Y. K., Ip, P. P., Huang, R.-L., Lai, H.-C., Cheung, A. N. Y., et al. (2018). Soluble E-Cadherin Promotes Tumor Angiogenesis and Localizes to Exosome Surface. Nat. Commun. 9 (1), 2270. doi:10.1038/s41467-01804695-7

Teleanu, R. I., Chircov, C., Grumezescu, A. M., and Teleanu, D. M. (2019). Tumor Angiogenesis and Anti-angiogenic Strategies for Cancer Treatment. Jcm 9 (1), 84. doi: $10.3390 / \mathrm{jcm} 9010084$

Todorova, D., Simoncini, S., Lacroix, R., Sabatier, F., and Dignat-George, F. (2017). Extracellular Vesicles in Angiogenesis. Circ. Res. 120 (10), 1658-1673. doi:10.1161/circresaha.117.309681

Treps, L., Perret, R., Edmond, S., Ricard, D., and Gavard, J. (2017). Glioblastoma Stem-like Cells Secrete the Pro-angiogenic VEGF-A Factor in Extracellular Vesicles. J. Extracellular Vesicles 6 (1), 1359479. doi:10.1080/ 20013078.2017.1359479

van Beijnum, J. R., Nowak-Sliwinska, P., Huijbers, E. J. M., Thijssen, V. L., and Griffioen, A. W. (2015). The Great Escape; the Hallmarks of Resistance to
Antiangiogenic Therapy. Pharmacol. Rev. 67 (2), 441-461. doi:10.1124/ pr.114.010215

Wang, F.-W., Cao, C.-H., Han, K., Zhao, Y.-X., Cai, M.-Y., Xiang, Z.-C., et al. (2019a). APC-activated Long Noncoding RNA Inhibits Colorectal Carcinoma Pathogenesis through Reduction of Exosome Production. J. Clin. Invest. 129 (2), 727-743. doi:10.1172/JCI122478

Wang, Y., Dong, L., Zhong, H., Yang, L., Li, Q., Su, C., et al. (2019b). Extracellular Vesicles (EVs) from Lung Adenocarcinoma Cells Promote Human Umbilical Vein Endothelial Cell (HUVEC) Angiogenesis through Yes Kinase-Associated Protein (YAP) Transport. Int. J. Biol. Sci. 15 (10), 2110-2118. doi:10.7150/ ijbs. 31605

Wang, Z.-F., Liao, F., Wu, H., and Dai, J. (2019c). Glioma Stem Cells-Derived Exosomal miR-26a Promotes Angiogenesis of Microvessel Endothelial Cells in Glioma. J. Exp. Clin. Cancer Res. 38 (1), 201. doi:10.1186/s13046-019-1181-4

Wang, H., Wang, L., Zhou, X., Luo, X., Liu, K., Jiang, E., et al. (2020a). OSCC Exosomes Regulate miR-210-3p Targeting EFNA3 to Promote Oral Cancer Angiogenesis through the PI3K/AKT Pathway. Biomed. Res. Int. 2020, 1-13. doi:10.1155/2020/2125656

Wang, M., Zhao, Y., Yu, Z.-Y., Zhang, R.-D., Li, S.-A., Zhang, P., et al. (2020b). Glioma Exosomal microRNA-148a-3p Promotes Tumor Angiogenesis through Activating the EGFR/MAPK Signaling Pathway via Inhibiting ERRFI1. Cancer Cel. Int. 20, 518. doi:10.1186/s12935-020-01566-4

Wang, Q., Wang, G., Niu, L., Zhao, S., Li, J., Zhang, Z., et al. (2021a). Exosomal MiR-1290 Promotes Angiogenesis of Hepatocellular Carcinoma via Targeting SMEK1. J. Oncol. 2021, 1-13. doi:10.1155/2021/6617700

Wang, Y., Cen, A., Yang, Y., Ye, H., Li, J., Liu, S., et al. (2021b). miR-181a, Delivered by Hypoxic PTC-Secreted Exosomes, Inhibits DACT2 by Downregulating MLL3, Leading to YAP-VEGF-Mediated Angiogenesis. Mol. Ther. - Nucleic Acids 24, 610-621. doi:10.1016/j.omtn.2021.02.027

Wu, F., Li, F., Lin, X., Xu, F., Cui, R.-R., Zhong, J.-Y., et al. (2019a). Exosomes Increased Angiogenesis in Papillary Thyroid Cancer Microenvironment. Endocr. Relat. Cancer 26 (5), 525-538. doi:10.1530/erc-19-0008

Wu, X.-G., Zhou, C.-F., Zhang, Y.-M., Yan, R.-M., Wei, W.-F., Chen, X.-J., et al. (2019b). Cancer-Derived Exosomal miR-221-3p Promotes Angiogenesis by Targeting THBS2 in Cervical Squamous Cell Carcinoma. Angiogenesis 22 (3), 397-410. doi:10.1007/s10456-019-09665-1

Xie, J.-y., Wei, J.-x., Lv, L.-h., Han, Q.-f., Yang, W.-b., Li, G.-1., et al. (2020a). Angiopoietin-2 Induces Angiogenesis via Exosomes in Human Hepatocellular Carcinoma. Cell Commun. Signal 18 (1), 46. doi:10.1186/s12964-020-00535-8

Xie, M., Yu, T., Jing, X., Ma, L., Fan, Y., Yang, F., et al. (2020b). Exosomal circSHKBP1 Promotes Gastric Cancer Progression via Regulating the miR-582$3 \mathrm{p} / \mathrm{HUR} / \mathrm{VEGF}$ axis and Suppressing HSP90 Degradation. Mol. Cancer 19 (1), 112. doi:10.1186/s12943-020-01208-3

Xu, Y., Leng, K., Yao, Y., Kang, P., Liao, G., Han, Y., et al. (2021). A Circular RNA, Cholangiocarcinoma-Associated Circular RNA 1, Contributes to Cholangiocarcinoma Progression, Induces Angiogenesis, and Disrupts Vascular Endothelial Barriers. Hepatology 73 (4), 1419-1435. doi:10.1002/hep.31493

Xuan, Z., Chen, C., Tang, W., Ye, S., Zheng, J., Zhao, Y., et al. (2021). TKI-resistant Renal Cancer Secretes Low-Level Exosomal miR-549a to Induce Vascular Permeability and Angiogenesis to Promote Tumor Metastasis. Front. Cel Dev. Biol. 9, 689947. doi:10.3389/fcell.2021.689947

Yan, W., Wang, Y., Chen, Y., Guo, Y., Li, Q., and Wei, X. (2021). Exosomal miR130b-3p Promotes Progression and Tubular Formation through Targeting PTEN in Oral Squamous Cell Carcinoma. Front. Cel Dev. Biol. 9, 616306. doi:10.3389/fcell.2021.616306

Yang, H., Zhang, H., Ge, S., Ning, T., Bai, M., Li, J., et al. (2018). Exosome-derived miR-130a Activates Angiogenesis in Gastric Cancer by Targeting C-MYB in Vascular Endothelial Cells. Mol. Ther. 26 (10), 2466-2475. doi:10.1016/ j.ymthe.2018.07.023

Yang, Y., Guo, Z., Chen, W., Wang, X., Cao, M., Han, X., et al. (2021). M2 Macrophage-Derived Exosomes Promote Angiogenesis and Growth of Pancreatic Ductal Adenocarcinoma by Targeting E2F2. Mol. Ther. 29 (3), 1226-1238. doi:10.1016/j.ymthe.2020.11.024

Yi, H., Ye, J., Yang, X.M., Zhang, L.W., Zhang, Z.G., and Chen, Y.P. (2015). HighGrade Ovarian Cancer Secreting Effective Exosomes in Tumor Angiogenesis. Int. J. Clin. Exp. Pathol. 8(5), 5062-5070.

Yin, H., Yu, S., Xie, Y., Dai, X., Dong, M., Sheng, C., et al. (2021). Cancer-associated Fibroblasts-Derived Exosomes Upregulate microRNA-135b-5p to Promote 
Colorectal Cancer Cell Growth and Angiogenesis by Inhibiting ThioredoxinInteracting Protein. Cell Signal. 84, 110029. doi:10.1016/j.cellsig.2021.110029

You, L.-N., Tai, Q.-W., Xu, L., Hao, Y., Guo, W.-J., Zhang, Q., et al. (2021). Exosomal LINC00161 Promotes Angiogenesis and Metastasis via Regulating miR-590-3p/ROCK axis in Hepatocellular Carcinoma. Cancer Gene Ther. 28 (6), 719-736. doi:10.1038/s41417-020-00269-2

Zeng, Z., Li, Y., Pan, Y., Lan, X., Song, F., Sun, J., et al. (2018). Cancer-derived Exosomal miR-25-3p Promotes Pre-metastatic Niche Formation by Inducing Vascular Permeability and Angiogenesis. Nat. Commun. 9 (1), 5395. doi:10.1038/s41467-018-07810-w

Zhang, L., Li, H., Yuan, M., Li, M., and Zhang, S. (2019). Cervical Cancer CellsSecreted Exosomal microRNA-221-3p Promotes Invasion, Migration and Angiogenesis of Microvascular Endothelial Cells in Cervical Cancer by Down-Regulating MAPK10 Expression. Cancer Manag. Res. 11, 10307-10319. doi:10.2147/cmar.S221527

Zhang, C., Luo, Y., Cao, J., Wang, X., Miao, Z., and Shao, G. (2020). Exosomal IncRNA FAM225A Accelerates Esophageal Squamous Cell Carcinoma Progression and Angiogenesis via Sponging miR-206 to Upregulate NETO2 and FOXP1 Expression. Cancer Med. 9 (22), 8600-8611. doi:10.1002/cam4.3463

Zheng, P., Luo, Q., Wang, W., Li, J., Wang, T., Wang, P., et al. (2018). Tumorassociated Macrophages-Derived Exosomes Promote the Migration of Gastric Cancer Cells by Transfer of Functional Apolipoprotein E. Cel Death Dis 9 (4), 434. doi:10.1038/s41419-018-0465-5

Zhou, X., Yan, T., Huang, C., Xu, Z., Wang, L., Jiang, E., et al. (2018). Melanoma Cell-Secreted Exosomal miR-155-5p Induce Proangiogenic Switch of CancerAssociated Fibroblasts via SOCS1/JAK2/STAT3 Signaling Pathway. J. Exp. Clin. Cancer Res. 37 (1), 242. doi:10.1186/s13046-018-0911-3

Zhou, Z., Zhang, H., Deng, T., Ning, T., Liu, R., Liu, D., et al. (2019). Exosomes Carrying microRNA-155 Target Forkhead Box O3 of Endothelial Cells and
Promote Angiogenesis in Gastric Cancer. Mol. Ther. - Oncolytics 15, 223-233. doi:10.1016/j.omto.2019.10.006

Zhuang, H., Wang, H., Yang, H., and Li, H. (2020). Exosome-encapsulated microRNA-21 from Esophageal Squamous Cell Carcinoma Cells Enhances Angiogenesis of Human Umbilical Venous Endothelial Cells by Targeting SPRY1. Cancer Manag. Res. 12, 10651-10667. doi:10.2147/ cmar.S259077

Ziaei, P., Geruntho, J. J., Marin-Flores, O. G., Berkman, C. E., and Grant Norton, M. (2017). Silica Nanostructured Platform for Affinity Capture of TumorDerived Exosomes. J. Mater. Sci. 52 (12), 6907-6916. doi:10.1007/s10853-0170905-0

Conflict of Interest: The authors declare that the research was conducted in the absence of any commercial or financial relationships that could be construed as a potential conflict of interest.

Publisher's Note: All claims expressed in this article are solely those of the authors and do not necessarily represent those of their affiliated organizations, or those of the publisher, the editors and the reviewers. Any product that may be evaluated in this article, or claim that may be made by its manufacturer, is not guaranteed or endorsed by the publisher.

Copyright (c) 2021 Huang, Lei, Zhong, Chung, Wang, Hu and Deng. This is an openaccess article distributed under the terms of the Creative Commons Attribution License (CC BY). The use, distribution or reproduction in other forums is permitted, provided the original author(s) and the copyright owner(s) are credited and that the original publication in this journal is cited, in accordance with accepted academic practice. No use, distribution or reproduction is permitted which does not comply with these terms. 